\title{
TITLE:
}

\section{Universal jump in the helicity modulus of the two-dimensional quantum XY model}

$\operatorname{AUTHOR}(S)$ :

Harada, Kenji; Kawashima, Naoki

\section{CITATION:}

Harada, Kenji ...[et al]. Universal jump in the helicity modulus of the two-dimensional quantum XY model. Physical Review B 1997, 55(18): R11949.

\section{ISSUE DATE:}

1997-05-01

URL:

http://hdl.handle.net/2433/200793

RIGHT:

(C) 1997 The American Physical Society 


\title{
Universal jump in the helicity modulus of the two-dimensional quantum $X Y$ model
}

\author{
Kenji Harada \\ Division of Applied Systems Science, Kyoto University, Sakyo-ku, Kyoto 606-01, Japan \\ Naoki Kawashima \\ Department of Physics, Toho University, Miyama 2-2-1, Funabashi 274, Japan
}

(Received 11 February 1997)

\begin{abstract}
The helicity modulus of the $S=1 / 2 X Y$ model is precisely estimated through a world-line quantum Monte Carlo method enhanced by a cluster update algorithm. The obtained estimates for various system sizes and temperatures are well fitted by a scaling form with $L$ replaced by $\log \left(L / L_{0}\right)$, which is inferred from the solution of the Kosterlitz renormalization group equation. The validity of the Kosterlitz-Thouless theory for this model is confirmed. [S0163-1829(97)50422-X]
\end{abstract}

The nature of the phase transition of the quantum $X Y$ model in two dimensions has not been fully clarified while the existence of a transition at a finite temperature was suggested $^{1,2}$ some years ago. Ding and Makivic ${ }^{3}$ examined this problem systematically by a large scale Monte Carlo simulation for the first time and concluded that a phase transition of the Kosterlitz-Thouless (KT) (Ref. 4) type takes place at $T_{\mathrm{KT}}=0.350(4)\left(\right.$ Ref. 3) or 0.353(3). ${ }^{5}$ In their calculation, the temperature dependence of the correlation length and the linear susceptibility were studied. However, it is technically difficult to distinguish an exponential divergence from an algebraic one. Because of this difficulty, the validity of their conclusion on the nature of the phase transition was questioned. ${ }^{6}$ For the same reason, Gupta and Baillie ${ }^{7}$ did not conclude, in spite of their very extensive Monte Carlo calculation, that the phase transition of the classical $X Y$ model is exactly what the KT theory predicts.

In previous studies, to our knowledge, a systematic study of the system size dependence of various quantities was missing for the quantum $X Y$ model. On the other hand, for other models with a KT phase transition, there are a number of reports on this size dependence. For example, Solýom and Ziman ${ }^{8}$ studied size dependence of the first excitation gap in the $S=1 / 2$ anisotropic $X X Z$ model in one dimension, which is exactly solvable and known to have a transition of the KT type at the antiferromagnetic isotropic point. They found that the exact estimates for finite systems do not fit into the standard form of the finite-size scaling at the critical point. Instead of using the ordinary finite-size scaling form, Weber and Minnhagen ${ }^{9}$ used the Kosterlitz renormalization group equation ${ }^{10}$ for the data analysis in their study of the classical $X Y$ model in two dimensions. They verified the KT-type phase transition by comparing the size dependence of the helicity modulus, $Y$, with the solutions of the renormalization group equation at the critical temperature. They found a remarkable agreement extending even to the prefactor of the logarithmic correction. Following the same idea, Olsson ${ }^{11}$ performed a more detailed analysis of the classical model with a more extensive Monte Carlo simulation. He found the resulting data including off-critical ones consistent with the Kosterlitz renormalization group equation.
The helicity modulus is known to exhibit the universal jump at the critical temperature. ${ }^{12}$ This quantity corresponds to the superfluid density when the model is regarded as a Boson system with hard cores. In the world-line quantum Monte Carlo method, ${ }^{13}$ the helicity modulus is related to the fluctuation in the total winding number of world lines by the following equation: ${ }^{14}$

$$
\Upsilon=(T / 2)\left\langle\boldsymbol{W}^{2}\right\rangle,
$$

where $\boldsymbol{W} \equiv\left(W_{x}, W_{y}\right)$ with $W_{x}\left(W_{y}\right)$ being the total winding number in the $x(y)$ direction. Makivic ${ }^{15}$ computed this quantity by means of a conventional world-line quantum Monte Carlo method. The method is not ergodic, ${ }^{16}$ however, in that it does not allow the winding number to vary. Therefore, Makivić resorted to an alternative definition of the quantity although it does not result in exactly the same answer as the conventional definition does. Another difficulty of the conventional Monte Carlo method is its long autocorrelation time near and below the critical temperature. These difficulties limited the accuracy and the precision of the Makivićs estimates and narrowed the temperature range of the simulation. To overcome these difficulties, we use the cluster Monte Carlo method ${ }^{16}$ in the present paper, in which both the number of particles and the winding number can vary. Another advantage of the method is its autocorrelation time which is often shorter than that of the conventional method by several orders of magnitude.

In the present paper, we attempt a detailed and precise comparison between the model and the theory by Kosterlitz ${ }^{10}$ through an accurate estimation of the thermal fluctuation in the winding number near and below the critical temperature. We will see that such an estimation allows us to examine a new scaling form which is different from the ordinary finitesize scaling. In this scaling form, the distance from the critical point, i.e., $K-K_{\mathrm{KT}}$ appears in the form of $(K$ $\left.-K_{\mathrm{KT}}\right)\left[\log \left(L / L_{0}\right)\right]^{2}$, in contrast to $\left(T-T_{\mathrm{KT}}\right) L^{y_{T}}$ in the ordinary finite-size scaling. At the same time, the quantity $x \equiv\left\langle(\pi / 2) \boldsymbol{W}^{2}\right\rangle-2$ scales as $x \log \left(L / L_{0}\right)$.

The $S=1 / 2$ quantum $X Y$ model is defined by the following Hamiltonian: 


$$
H=-J \sum_{\langle i j\rangle}\left(S_{i}^{x} S_{j}^{x}+S_{i}^{y} S_{j}^{y}\right),
$$

where $\langle i j\rangle$ runs over all nearest-neighbor pairs on the square lattice. As for the spin operators, we here use the convention in which $\left(S_{i}^{\mu}\right)^{2}=1 / 4(\mu=x, y, z)$.

In comparing the numerical result with the renormalization group theory, the squared winding number is a useful quantity since it can be regarded as the renormalized coupling constant and therefore its size dependence can be directly predicted by the following renormalization equation: ${ }^{10}$

$$
\frac{d x}{d l}=-y^{2}, \quad \frac{d y}{d l}=-x y .
$$

Here, $x$ and $y$ are renormalized parameters after a renormalization operation up to the length scale $L \equiv L_{0}(T) e^{l}$ where $L_{0}(T)$ is some characteristic length of the order of the lattice constant and has no singularity at $T=T_{\mathrm{KT}}$. The parameter $x$ is related to the renormalized coupling constant, i.e., the helicity modulus, by the following equation: ${ }^{12}$

$$
x=\frac{\pi \Upsilon}{T}-2=\frac{\pi}{2}\left\langle W^{2}\right\rangle-2 .
$$

In what follows, we regard the estimate of $(\pi / 2)\left\langle W^{2}\right\rangle-2$ for a system of the size $L$ at the temperature $T$ as $x\left(l=\log \left[L / L_{0}(T)\right], T\right)$.

Equation (3) has an integral

$$
\Delta(T) \equiv x^{2}(l)-y^{2}(l)
$$

which does not depend on $l \equiv \log \left[L / L_{0}(T)\right]$. As a function of $T$, this integral is nonsingular at $T=T_{\mathrm{KT}}$, i.e., $\Delta(K)=a\left(K-K_{\mathrm{KT}}\right)+b\left(K-K_{\mathrm{KT}}\right)^{2}+\cdots \quad$ where $\quad K \equiv J / T$. The solution of Eq. (3) is given by

$$
x(T, L)= \begin{cases}\sqrt{|\Delta|} \operatorname{coth}(\sqrt{|\Delta|} l) & \left(K>K_{\mathrm{KT}}\right) \\ \sqrt{|\Delta|} \cot (\sqrt{|\Delta|} l) & \left(K<K_{\mathrm{KT}}\right) .\end{cases}
$$

Note that the ordinary finite-size scaling form

$$
x(T, L)=L^{\omega} g\left(\Delta L^{1 / \nu}\right)
$$

cannot be consistent with Eq. (6). Instead, the solution (6) is a special case of the following form that one can obtain from the ordinary finite-size scaling form by replacing $L$ by $l=\log \left[L / L_{0}(T)\right]$,

$$
x(T, L)=l^{-1} f\left(\Delta l^{2}\right) .
$$

When expressed in the form of Eq. (8), the solution (6) corresponds to a "scaling" function $f(X)$ which is nonsingular at $X=0$ in spite of the singularity in $\sqrt{|\Delta|}$ at $T=T_{\mathrm{KT}}$. To be more specific, the solution (6) corresponds to

$$
f(X)=1+O(X) .
$$

Note that the fitting functions used by Olsson ${ }^{11}$ [Eqs. (11a)(11c) with (16) and (18) in his paper] are consistent with this scaling form. Olsson's fitting function corresponds to ours with a temperature-independent $L_{0}(T)$.

For our Monte Carlo simulation, using Suzuki-Trotter decomposition, we transformed the two-dimensional quantum
$X Y$ model into the $(2+1)$-dimensional Ising model with four spin plaquette interactions. The partition function is described as

$$
Z=\sum_{S} \prod_{p} \omega\left(S_{p}\right),
$$

where $S$ is the set of states $S_{i, t}^{z}$ on the (2+1)-dimensional lattice, $S_{p}$ is a state of spins in a plaquette $p$ formed by four spins $S_{i, t}^{z}, S_{j, t}^{z}, S_{i, t+1}^{z}, S_{j, t+1}^{z}$, and $\omega\left(S_{p}\right)$ is the two-spin propagator defined below. We apply periodic boundary conditions in all directions to preserve the translational invariance and to satisfy the trace requirements.

By numbering four local states on a bond as $1=(\uparrow \uparrow)$, $2=(\uparrow \downarrow), 3=(\downarrow \uparrow)$, and $4=(\downarrow \downarrow)$, the two-spin propagator can be written explicitly as

$$
\omega=\left(\begin{array}{cccc}
1 & 0 & 0 & 0 \\
0 & \cosh \left(\frac{\Delta \tau}{2}\right) & \sinh \left(\frac{\Delta \tau}{2}\right) & 0 \\
0 & \sinh \left(\frac{\Delta \tau}{2}\right) & \cosh \left(\frac{\Delta \tau}{2}\right) & 0 \\
0 & 0 & 0 & 1
\end{array}\right),
$$

where $\Delta \tau=J /\left(m k_{\mathrm{B}} T\right)$ with the Trotter number $m$, and the temperature $T$. In what follows, we will set $J=1$ and $k_{\mathrm{B}}=1$ for simplicity.

We have carried out a Monte Carlo simulation using a cluster algorithm called the loop algorithm. ${ }^{16}$ In this algorithm, four spins in each plaquette are connected pairwise. The pairs are chosen stochastically. The connected spins form loops which are units of flipping. A world line is a path connecting sites with up spins which may wind around the system with periodic boundary conditions. The winding numbers $W_{x}$ (or $W_{y}$ ) are defined as the sum of the numbers of such windings of all world lines in the $x$ (or $y$ ) direction. For example, $W_{x}$ can be rewritten as

$$
W_{x}=\frac{1}{L_{x}} \sum_{p} \alpha_{x}\left(S_{p}\right),
$$

where $L_{x}$ is the lattice size in the $x$ direction. The symbol $\alpha_{x}\left(S_{p}\right)$ stands for the function which takes the value 1 (or -1 ) if a world line passes through the plaquette $p$ in the positive (or negative) $x$ direction and the value 0 , otherwise. The other winding number $W_{y}$ is defined in the same manner.

In order to reduce the statistical error, we have used an improved estimator for the squared winding number of world lines. It simply equals one-fourth of the sum of squared winding numbers of loops formed by a clustering procedure (i.e., a graph assignment procedure) in the cluster Monte Carlo method. A more detailed discussion will be given elsewhere. ${ }^{17}$ In the present simulation, we have found that the new estimator reduces errors by about twenty percent.

In our simulations, we have taken various temperatures between 0.22 and 0.60 and used lattices with $L=8,16,32$, and 64 . As the Trotter number $m$, we have used $m=8,16$, and 32 . When the systematic error due to the Trotter discretization exceeds the statistical error, we have 


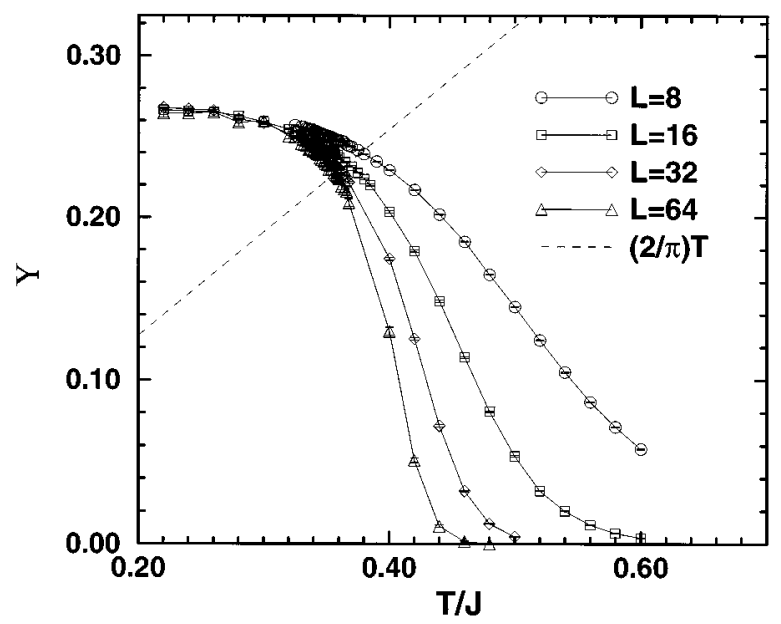

FIG. 1. The helicity modulus (or superfluid density) $Y=(T /$ 2) $\left\langle\mathbf{W}^{2}\right\rangle$ as a function of temperature. The universal jump is expected at the point where $Y=2 T / \pi$. Error bars are drawn but most of them are so small that they cannot be recognized.

reduced the systematic error by the extrapolation to $m=\infty$ using three data for different Trotter numbers. The length of a typical run on $L=64$ at a temperature is more than $2 \times 10^{5}$ Monte Carlo Sweeps (MCS). To make use of the vector processors, we have developed the efficient vectorized code for the cluster identification. The underlying idea for this vectorization was proposed by Mino ${ }^{18}$ and it is based on the "divide-and-conquer" strategy. In his algorithm, one firstly divides the lattice into small sublattices and, at the same time, identifies clusters in each sublattice neglecting the connectivity outside of the sublattice. One then deals with twice as large sublattices formed by a pair of previous sublattices. By repeating this procedure, one can eventually get clusters identified correctly in the whole lattice. Using this algorithm, we have achieved the efficiency of 1.5 million site updates per second on the Fujitsu's VPP500.

Each run is divided into several bins. The length of a bin is taken so that bin averages are statistically independent from each other at least approximately. We have measured integrated autocorrelation times for $\boldsymbol{W}^{2}$ at low temperatures

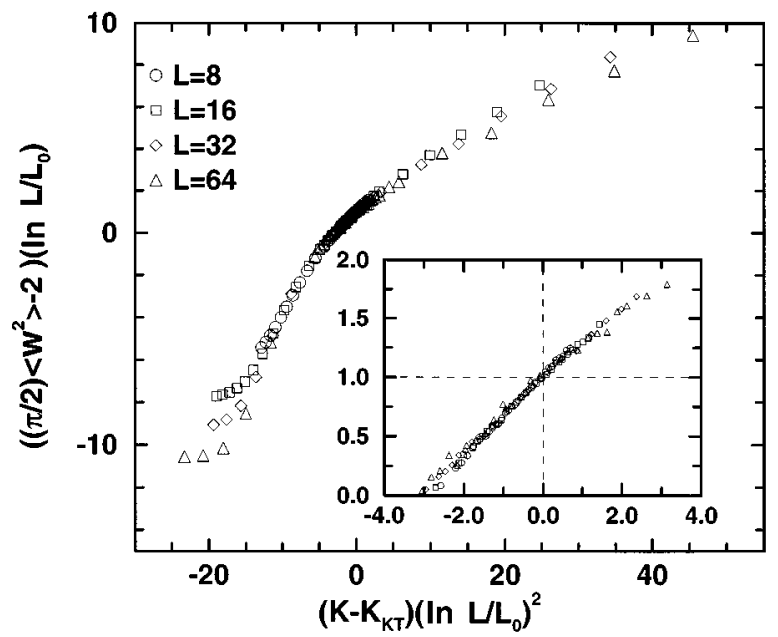

FIG. 2. A rescaled plot of the winding number fluctuation. The inset is an enlarged view of the critical point.

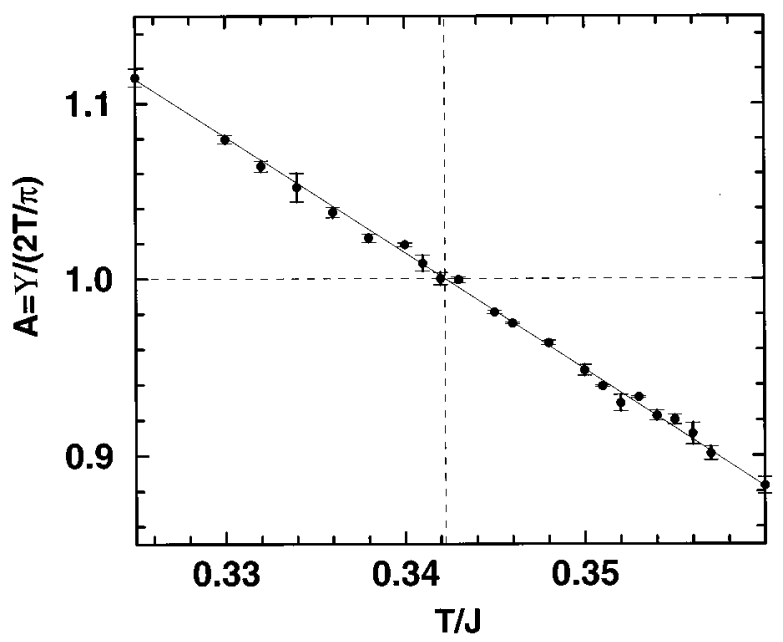

FIG. 3. The helicity modulus divided by the magnitude of its universal jump. The dashed vertical line indicates the critical temperature at which the solid line crosses the dashed horizontal line $(A=1)$. The solid line is determined by a linear fitting.

$T<0.35 \mathrm{~J}$ by the standard binning analysis for a run of $10^{5}$ MCS with $L=64$. They turn out to be smaller than 2 MCS in all cases. Since the smallest bin length used in the main calculation is 1500 MCS, the statistical independence among bins is assured. The statistical errors of various observables have been calculated from the standard deviation of the binaverage distribution.

In Fig. 1, the raw data for $\left\langle\boldsymbol{W}^{2}\right\rangle \equiv\left\langle W_{x}^{2}+W_{y}^{2}\right\rangle$ are plotted. Figure 2 is its "scaling plot" using the the form of Eq. (8) assuming that $L_{0}(T)$ does not depend on the temperature, which was a reasonably good approximation judging from Olsson's observation ${ }^{11}$ (see Fig. 14) for the classical model. $\left[L_{0}(T)\right.$ in the present paper corresponds to "const" in Eq. (18) in Olsson's paper.] The enlarged view around the critical point is shown as the inset. The scaling plot in Fig. 2 is a reasonably good one, considering the fact that we have only two adjustable parameters, $K_{\mathrm{KT}}$ and $L_{0}$, while there are three such parameters in the ordinary finite-size scaling plot. The value of $K_{\mathrm{KT}}$ is determined so that the resulting plot gives the "best" scaling plot. To quantify the "goodness" of the scaling plot, a cost function $S\left(K_{\mathrm{KT}}, L_{0}\right)$ is defined, ${ }^{19}$ which is essentially the deviation from the local linear fitting. We used the data in the range $0<\left\langle\boldsymbol{W}^{2}\right\rangle-4 / \pi<0.25$, which roughly corresponds to the temperature range $0.330<T<0.375$. The best fitting is obtained with the transition temperature

$$
T_{\mathrm{KT}}=J / K_{\mathrm{KT}}=0.342(2) \mathrm{J}
$$

which is significantly lower than Ding and Makivić's estimates. ${ }^{3,5}$ We confirmed that other choices of the range result in the estimates of the critical temperature consistent with the value quoted above. The best scaling plot is shown in Fig. 2. It should be noted that the estimated scaling function takes the value of 1 at $K-K_{\mathrm{KT}}=0$ as is predicted by the Kosterlitz renormalization group equation [see Eq. (9)]. Of course, an ordinary finite-size scaling does not predict the value of the scaling function at this particular point. Considering the fact that we have not used this information while making the scaling plot, the agreement between the scaling 
theory and the numerical result is hardly understandable unless we assume the validity of the KT theory. (Note that the criterion we have adopted in choosing the value of $K_{\mathrm{KT}}$ is simply that all points should fall onto a single curve.) Therefore, we consider that the present result is a strong confirmation of the validity of Kosterlitz's scaling theory in the quantum $X Y$ model in two dimensions.

We have also tried another analysis following Weber and Minnhagen. ${ }^{9}$ At each temperature, we assumed the following system size dependence of the helicity modulus:

$$
\frac{\pi \Upsilon}{2 T}=\frac{\pi}{4}\left\langle\boldsymbol{W}^{2}\right\rangle=A(T)\left(1+\frac{1}{2 \log \left[L / L_{0}(T)\right]}\right) .
$$

This fitting form is correct at the critical point with $A\left(T_{\mathrm{KT}}\right)=1$ [Eqs. (8) and (9)]. Since the number of data at each temperature is small, the critical temperature cannot be determined as the one at which the fitting is best. Instead, we plot the coefficient $A(T)$ as a function of temperature (Fig. $3)$. The critical temperature, then, is estimated as the point at which $A(T)$ takes on the value 1 . Linear fitting of the data yields

$$
T_{\mathrm{KT}}=0.3423(3) \mathrm{J},
$$

which is consistent with Eq. (13). [It is natural that here we have a more precise value than Eq. (13), since we have assumed Eq. (14), a more specific fitting function than Eq. (8).]

To summarize, the loop algorithm has proven to be very efficient in studying the winding number particularly at low temperatures, and we have confirmed that the phase transition in the quantum $S=1 / 2 X Y$ model is of the KT type avoiding the subtle and technically difficult comparison between an exponential divergence and an algebraic one. We have demonstrated that not only the magnitude of the jump but also very fine points in the Kosterlitz-Thouless-Nelson theory are realized in the present model.

The authors would like to thank K. Nomura for useful comments and discussions. The computations in the present work were performed on Fujitsu's VPP500 at Kyoto University Data Processing Center and partially at ISSP, the University of Tokyo.
${ }^{1}$ E. Loh, Jr., D. J. Scalapino, and P. M. Grant, Phys. Rev. B 31, 4712 (1985).

${ }^{2}$ T. Onogi, Ph.D. thesis, University of Tokyo, 1986.

${ }^{3}$ H.-Q. Ding and M. S. Makivić, Phys. Rev. B 42, 6827 (1990); M. S. Makivić and H.-Q. Ding, ibid. 43, 3562 (1991).

${ }^{4}$ J. M. Kosterlitz and D. J. Thouless, J. Phys. C 6, 1181 (1973).

${ }^{5}$ H.-Q. Ding, Phys. Rev. B 45, 230 (1992).

${ }^{6}$ J. Sznajd, Phys. Rev. B 45, 489 (1992); H.-Q. Ding and M. S. Makivić, ibid. 45, 491 (1992).

${ }^{7}$ R. Gupta, J. DeLapp, G. G. Batrouni, G. C. Fox, C. F. Baillie, and J. Apostolakis, Phys. Rev. Lett. 61, 1996 (1988); R. Gupta and C. F. Baillie, Phys. Rev. B 45, 2883 (1992).

${ }^{8}$ J. Sólyom and T. A. L. Ziman, Phys. Rev. B 30, 3980 (1984).

${ }^{9}$ H. Weber and P. Minnhagen, Phys. Rev. B 37, 5986 (1987).

${ }^{10}$ J. M. Kosterlitz, J. Phys. C 7, 1046 (1974).
${ }^{11}$ P. Olsson, Phys. Rev. B 52, 4526 (1995).

${ }^{12}$ D. R. Nelson and J. M. Kosterlitz, Phys. Rev. Lett. 39, 1201 (1977).

${ }^{13}$ See, for example, Monte Carlo Methods in Equilibrium and Nonequilibrium Systems, edited by M. Suzuki (Springer, Berlin, 1987).

${ }^{14}$ E. L. Pollock and D. M. Ceperley, Phys. Rev. B 36, 8343 (1987).

${ }^{15}$ M. S. Makivić, Phys. Rev. B 46, 3167 (1992).

${ }^{16}$ H. G. Evertz, M. Marcu, and G. Lana, Phys. Rev. Lett. 70, 875 (1993); N. Kawashima, J. Stat. Phys. 82, 131 (1996), and references there.

${ }^{17}$ K. Harada and N. Kawashima (unpublished).

${ }^{18}$ H. Mino, Comput. Phys. Commun. 66, 25 (1991).

${ }^{19}$ N. Kawashima and N. Ito, J. Phys. Soc. Jpn. 62, 435 (1993). 